\title{
An inferential comparison between the capabilities and achievements of 1 st-year medical and nursing students at the University of the Free State, Bloemfontein, South Africa
}

\author{
A M Gerber, ${ }^{1} \mathrm{PhD} ; \mathbf{R}$ Botes, ${ }^{2} \mathrm{MSc} ;$ A Vorster, ${ }^{1} \mathrm{MB} \mathrm{ChB}$ \\ ${ }^{1}$ Department of Basic Medical Sciences, Faculty of Health Sciences, University of the Free State, Bloemfontein, South Africa \\ ${ }^{2}$ Department of Clinical Epidemiology, University Medical Center Groningen, University of Groningen, The Netherlands
}

Corresponding author: A Gerber (gerberam@ufs.ac.za)

Background. Research indicates that academic stressors, living circumstances, working conditions and where students undertake leisure activities affect academic performance, capabilities and achievements (functionings).

Objective. To investigate how 1st-year medical and nursing students perceived their own capabilities compared with their actual achievements (functionings). The article focuses on the achievements (functionings), as these students were admitted through a selection process, indicating their potential capability to succeed.

Methods. In this descriptive, comparative study, all 1st-year medical and nursing students at the University of the Free State, Bloemfontein, South Africa were invited to complete a validated questionnaire to reflect their capabilities (scope) and achievements (outcomes). The questionnaire incorporated seven domains: happiness, achievements, health, intellect, social relations, environment and integrity. Data were analysed using descriptive statistics (frequencies, medians, means, standard deviations and standard errors).

Results. All respondents valued the domains positively with regard to the outcomes (functionings). On average, nursing students valued the domains $17.4 \%$ lower than the medical students. Integrity was valued the highest by all. Health scored the lowest in the medical group, and environment (where students study and undertake leisure activities) the lowest in the nursing group.

Conclusions. Medical schools should include wellness in their curricula, limit the degree of physical and emotional exhaustion associated with training, and have realistic expectations of students. Programmes should allocate enough time for students to manage their time well to take part in physical activity and eat healthy foods. Nursing students' work environment should improve. More time should be made available for leisure activities and improvement to students' study environment.

Afr J Health Professions Educ 2016;8(2):200-202. DOI:10.7196/AJHPE.2016.v8i2.719

Research has shown that academic stressors, living circumstances, working conditions and where students undertake their leisure activities affect their academic performance, capabilities and functionings. At a tertiary educational level, individuals are exposed to academic material and activities that should promote basic capabilities and functionings as well as academic achievement. ${ }^{[1]}$ Amartya Sen proposed the capability approach, which is a philosophical perspective that has grown into an interdisciplinary paradigm for development and wellbeing on an educational, personal and social level. ${ }^{[2]}$

The capability approach is a multifactorial concept that reflects on people's capabilities and functionings. This concept supports the ideas of people and the scope of their abilities to achieve, while successes are the actual achievements they accomplish. Human advancement and development can only occur when capabilities and achievements are realistic and attainable. $^{[3]}$ The term capability can encompass skills, attributes and competences. Stephenson and Yorke ${ }^{[4]}$ define capability as 'an integration of knowledge, skills, personal qualities and understanding, used appropriately and effectively in response to new and changing circumstances. Capability can be observed while watching people act with confidence in their ability to: (i) explain what they stand for; (ii) live and work effectively with others; (iii) take effective and appropriate action; and (iv) continue to learn from their experiences as individuals and in association with others in a diverse and changing society. Each of these four abilities is a complex integration of different skills and qualities. Capable people are not only proficient in their areas of specialty, but also have the confidence to apply their knowledge and skills in varied and changing situations, and continue to develop their specialist knowledge and skills long after they have left formal education. Capability connotes integration, lifelong learning and the confidence to realise future potential in a developmental and self-managed way. ${ }^{[4]}$

It has been suggested that a beneficial advancement of personal abilities and achievements occur through education and learning. ${ }^{[5,6]}$ According to Saito, ${ }^{[7]}$ there is a potentially strong and mutually enhancing relationship between the capability approach and education. These capabilities and functionings include happiness, satisfying social relations, personal integrity and a healthy life that needs to be advanced to create a successful learning environment. ${ }^{[5,6]}$ It would be reasonable to assume that the educational process leads to heightened consciousness, self-enhancement, empowerment and informed choices in both academic and personal life. ${ }^{[6,8]}$

The strength of the capability approach lies in its capacity to provide sensible tools and frameworks, within which literacy, competencies and other educational aspects might be conceptualised and evaluated using a validated questionnaire.

\section{Objective}

Our study focused on the academic capabilities and achievements of 1st-year medical and nursing students. We investigated how students perceived their personal scope of abilities (capabilities) compared with 
their successes (functionings) in the different domains. The article focuses on the achievements of students, based on admission to a tertiary institution by means of a selection process, who were expected to have the ability to succeed and achieve more compared with the general students admitted to tertiary institutions.

\section{Methods}

This was a descriptive, comparative study with quantitative elements. All 1st-year medical and nursing students at the University of the Free State, Bloemfontein, South Africa were invited to complete a validated questionnaire to reflect their capabilities (scope) and achievements (outcomes). Questionnaires were distributed during lectures. Participation was voluntary and, as no identifiable information was captured, anonymity was ensured. Students were informed about the context of the study and that completion of the questionnaire would take $\sim 15$ minutes.

\section{The questionnaire}

The standardised Capabilities and Achievements Questionnaire, validated by Anand, was selected for this study. ${ }^{[5]}$ Seven different domains were identified and include happiness, achievements, health, intellect, social relations, environment and integrity. Respondents were able to give their perceptions of their personal successes by guided options in the questionnaire. The first section investigated the scope of abilities (capabilities) each respondent felt that they were capable of achieving, by rating their abilities on a Likert scale from 1 (very good) to 7 (very inadequate). The next section required the respondents to reflect on the outcomes (successes) they were able to achieve in their lives. They had to rate the outcomes on a Likert scale from 1 (strongly agree) to 7 (strongly disagree).

\section{Ethical considerations}

A protocol was approved by the Ethics Committee, Faculty of Health Sciences, University of the Free State (ECUFS 105/2011). Permission to perform the survey was obtained from the vice-rector: Academic Planning, and the dean of the Faculty of Health Sciences, University of the Free State.

\section{Data management and statistical analysis}

The data were analysed and interpreted by the investigators and a statistician. Data are presented by standard descriptive statistics (frequen- cies, medians, means, standard deviations and standard errors).

Trends, correlations and meaningful differences were noted, from which consequential conclusions were drawn. As the students would reflect their perceptions/opinions with regard to their own functionings, as defined by the questionnaire, implicit weight was attributed to each of the domains. The results of the seven domains regarding scope of abilities and outcomes were grouped together according to the responses: positive (1 - 3), neutral (4), or negative (5 - 7).

\section{Results}

Respondents ( $N=148$; 68 medical and 80 nursing students) completed the questionnaire. Fig. 1 shows the results of the different domains when comparing the two groups. Both the medical and nursing students rated the domains on integrity and intellect the highest. The medical students valued all the domains positively $(>60 \%)$ in terms of the outcomes. On average, the nursing students valued all the outcomes $17.4 \%$ lower than the medical students, the greatest difference (30\%) being for the environment domain. Medical students rated achievement (75\%) and health (69\%) as the domains they were least satisfied with. Nursing students indicated that they were least satisfied with their environment (48\%) and health (53\%).

The medical students' academic performance indicated that $32 \%$ achieved marks between $51 \%$ and $60 \%$, and $66 \%$ achieved $>60 \%$ in their respective tests for the semester in which the survey was done.

Nursing students achieved a pass rate of $34 \%$, with marks between $51 \%$ and $60 \%$, while $53 \%$ attained a $>60 \%$ mark.

\section{Discussion}

Our findings indicate that the 1st-year medical and nursing students valued integrity and intellectual outcomes the highest. This might in part be owing to these students being in an intellectually stimulating and challenging environment, with extensive focus on integrity and ethical behaviour.

As for the health domain - rated worst by the medical group and second worst by the nursing group - it is clear that both groups were not satisfied with factors regarding their personal health. Insufficient sleep, mild exhaustion, poor eating habits and little time to exercise may be contributing factors.

The results further showed that medical students rated their achievements notably higher than nursing students. However, the achievement domain was still rated second lowest in the medical group, reflecting their opinion that they should perform better, which could be a stressor in their lives. Even though they value achievement, they are not satisfied with their personal level of achieving success. This may also contribute to their poor validation of personal health due to stress with regard to successful outcomes. For the nursing students, time management could have been a contributing factor to their poorer academic achievements, as they have to work 440 clinical hours per year as part of their training.

The nursing students indicated their environment domain as the worst. They rated their living circumstances, working conditions and where they undertake leisure activities as unsatisfactory or not ideal.

Comparing our nursing group results with findings reported by Pryjmachuk and Richards, ${ }^{[9]}$ the three main group stressors that they identified

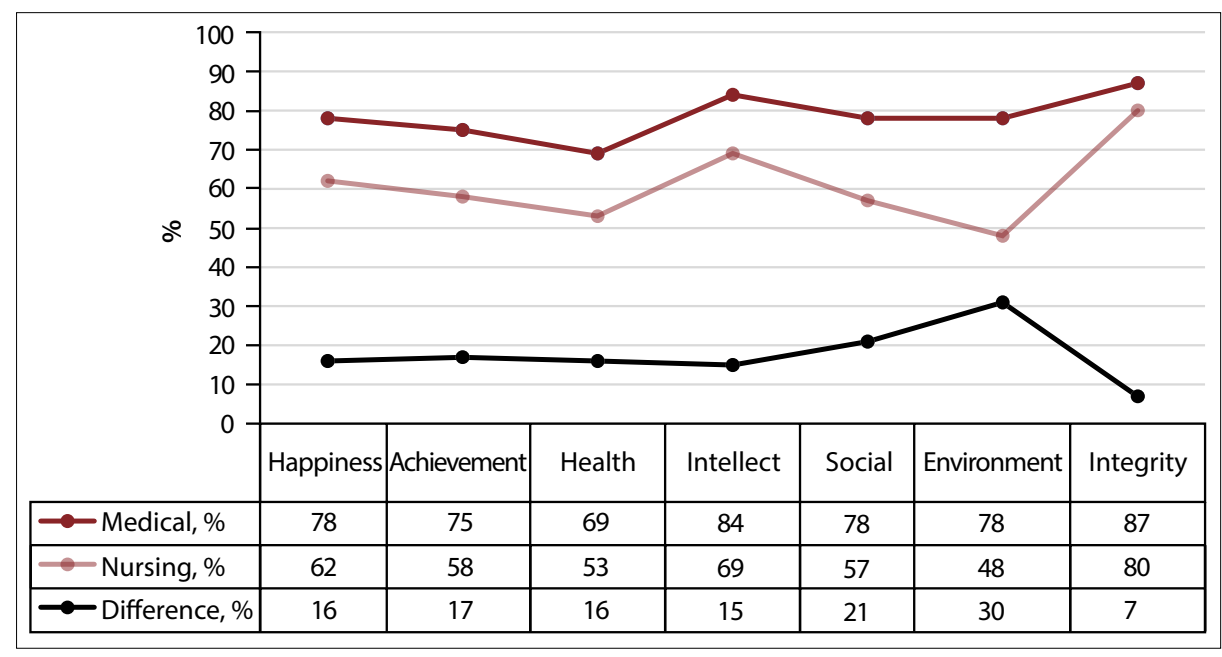

Fig. 1. The percentage of 1st-year medical and nursing students that rated each of the seven domains as positive. 
were similar to our results. These include: $(i)$ academic stressors (testing and evaluation, fear of failure with regard to training, workload); (ii) clinical stressors (work, fear of making mistakes, negative responses to death or patients' suffering); and (iii) personal/social stressors (economic problems, imbalance between household and academic work). ${ }^{[9]}$

A similar study also reported a diversity of stress sources, and that high levels of stress were associated with poor academic performance. These stressors were identified as normal stressors in day-to-day living, additional stress of the course workload, lack of leisure time, material to be learnt, and frequent academic examinations in a competitive environment. ${ }^{[10]}$ Our results were similar to these.

The capability approach has many facets, which reflect upon people's capabilities and functionings. Human advancement is needed to develop ability and achieve successes. Students need self-discipline, support from home, ability to work independently, resilience, and relevance of their skills to future work, i.e. characteristics a 1st-year student needs to be successful in life. Some of these characteristics are not evident in all 1st-year students and need to be developed and improved. ${ }^{[1]}$

\section{Conclusions}

The students in both groups indicated integrity and intellect as the domains with which they were most satisfied. Both the medical and nursing students indicated a negative response in the health domain. They were concerned about their eating habits and time spent on physical activities. The achievement domain was perceived unsatisfactorily among the medical students. They were of the opinion that they could achieve better academically and perform better. The nursing students experienced their working environment, where they undertake social activities, and study environment as unsatisfactory. To address these challenges, it is suggested that faculties of health sciences should introduce wellness curricula, set realistic expectations for the workload, and limit the degree of physical and emotional exhaustion associated with training. Successful role-models or mentors should be provided to assist students. Giving students the opportunity to be responsible and accountable for their own learning may result in better performance in their working and personal lives. This would promote a deeper understanding of their course content, build confidence in their ability to learn, and develop better skills across various functional, personal, social and professional platforms.

Tertiary institutions should direct more effort towards creating circumstances where students can experience joy and satisfaction. This could include conditions that promote studying in a pleasant environment, provision of financial aid, if necessary, provision of adequate resources, and general support. Time management also includes time set aside for satisfying social relationships and extramural physical activities.

Supporting students on an academic and personal developmental level may result in a better quality of life with improved academic performance and a more capable student, which translates into more competent and capable doctors and nurses for the future.

Capabilities and Achievements Questionnaire. This questionnaire is available from the corresponding author on request.

Acknowledgement. We thank Ms T Mulder, medical editor, School of Medicine, University of the Free State, for technical and editorial preparation of the manuscript.

\section{References}

1. Dyrbye LN, West CP, Satele D, et al. Burnout among US medical students, residents, and early career physicians relative to the general US population. Acad Med 2014;89(3):443-451. DOI:10.1097/acm.0000000000000134

2. Martinetti E. A multidimensional assessment of well-being based on Sen's functioning approach. http://www-3 unipvit/cds/userfiles/file/Papers/paper_chiappero_1 pdf (accessed 24 June 2016).

3. Krishnakumar J. Going beyond functionings to capabilities: An econometric model to explain and estimate capabilities. J Hum Dev 2007;8(1):39-63. DOI:10.1080/14649880601101408

4. Stephenson J, Yorke M. Capability and Quality in Higher Education. London: Kogan Page, 1998

5. Anand P, van Hees M. Capabilities and achievements: An empirical study. J Socio-Economics 2006;35(2):268284. DOI:10.1016/j.socec.2005.11.003

6. Young M. Basic capabilities, basic learning outcomes and thresholds of learning. J Hum Dev Capab 2009;10(2):259-277. DOI: $10.1080 / 19452820902941206$

7. Saito M. Amartya Sen's capability approach to education: A critical exploration. J Philos Educ 2003;37(1):17-33. DOI: $10.1111 / 1467-9752.3701002$

8. Robeyns I.The capability approach:A theoretical survey.JHum Dev 2005;6(1):93-117.DOI:10.1080/146498805200034266

9. Pryjmachuk S, Richards DA. Mental health nursing students differ from other nursing students: Some observations from a study on stress and coping. Int J Ment Health Nurs 2007;16(6):390-402. DOI:10.1111/1.1447-
of observations from

10. Sohail N. Stress and academic performance among medical students. J Coll Physicians Surg Pak 2013;23(1):67-71.

11. Naong N, Zwane G, Mogashoa G, Fleischmann E. Challenges of teaching first-year students at institutions of higher learning. http://www.ccsenet.org/journal/index.php/ies/article/view/1719 (accessed 24 June 2016). 